\title{
PROGRAMACIÓN ATRACTIVA DE PLC
}

\author{
Eduardo J. Moya de la Torre \\ ITAP, Universidad de Valladolid, 47011, Valladolid, edumoy@eii.uva.es \\ Alfonso Poncela, F. Javier García Ruiz \\ ITAP, Universidad de Valladolid, 47011, Valladolid \{poncela,javgar\}@eii.uva.es \\ Víctor del Barrio Lagándara, victor.barrio.lagandara@alumnos.uva.es
}

\begin{abstract}
El objetivo de este artículo es presentar una nueva forma de transmitir los conocimientos en cuanto al manejo y programación de autómatas se refiere, utilizando para ello un software de simulación que nos permita comprobar en todo momento cual sería el resultado físico de las instrucciones programadas en el autómata. Se mostrará su funcionamiento con fines puramente didácticos.
\end{abstract}

Se recurre al diseño y programación de varias estaciones de trabajo que se asemejen en gran medida muchos de los procesos que se dan hoy en día a nivel industrial.

El equipo necesario para llevar a cabo esta tarea consta de una parte software compuesta por el programa de simulación (FACTORY I/O) y el programa para realizar la programación de las diferentes instrucciones del autómata (TIA PORTAL), así como de una parte hardware en la que se encuentran elementos como el ordenador con el que se realizará la programación de los diferentes elementos y se simulará la estación, un PLC encargado de gobernar el control de nuestras plantas virtuales y una pantalla HMI que nos permite monitorizar alguno de los procesos.

Palabras Clave: PLC, simulación, TIA PORTAL, FACTORY I/O, programación.

\section{INTRODUCCIÓN}

En el marco de las asignaturas de control que se imparten sobre todos en el Grado de Electrónica Industrial y Automática (GEIA) en la Universidad de Valladolid, se desea realizar un conjunto de prácticas que sirva como apoyo a otros equipos de prácticas, con un manejo simple y fácilmente transportable.

El creciente desarrollo de la automatización de procesos que se ha dado en estos últimos años hace que cada vez adquiera más relevancia por universidades e instituciones educativas la impartición de conocimientos asociados con la robótica, el control o la instrumentación.

El problema que se presenta en la mayoría de las ocasiones es que este tipo de conocimientos poseen una gran carga matemática que hacen que la asimilación de conceptos sea un tanto compleja y abstracta, dificultando la interiorización de los mismos.

Por otro lado, la impartición de dichos conocimientos de manera individualizada muchas veces no permite ver la relación que existen entre ellos ya que al final unos se apoyan en las funciones de otros para poder trabajar.

Todos estos problemas se solucionan cuando el estudiante una vez finalizado sus estudios accede a un puesto de trabajo y ve en la realidad como en una máquina de un proceso industrial están todos los conceptos que había estudiado, pero sin obtener una visión global de los mismo.

Para los centros educativos proporcionar dichas oportunidades es casi imposible debido al elevado coste económico que poseen las maquinas a nivel industrial. Sin embargo, hoy en día existen softwares de simulación y de programación que aúnan gran parte de los conocimientos teóricos y que permiten visualizar como sería el comportamiento real de los mismos, tal y como si estuviéramos en una planta real, permitiendo al alumno obtener una visión global de la automatización de procesos.

El presente artículo se organiza de la siguiente manera: después de esta breve introducción, en la segunda sección se muestran los objetivos principales del trabajo, en la tercera sección se hace una descripción del software de simulación FACTORY I/O así como de sus principales características y librerías. En la cuarta sección se presentan los elementos necesarios para el desarrollo de las estaciones creadas en la aplicación. En la quinta sección se presentan cada una de las cuatro estaciones de trabajo creadas con su diseño, conexionado, funcionamiento y programación a partir de los elementos básicos, Finalmente, se ofrecen algunas de las principales conclusiones obtenidas para la utilización del equipo de prácticas en el Grado de Electrónica Industrial y Automática (GEIA) en la Universidad de Valladolid, y las referencias utilizadas. 


\section{INTRODUCCIÓN}

El objetivo del presente trabajo es la programación y simulación de cuatro procesos industriales en los que se manejarán tanto señales analógicas como digitales, se regularán procesos continuos mediante control PID además de monitorizar algunas acciones por pantalla HMI.

El hardware empleado es un ordenador personal, un autómata S7-1200 de Siemens y una pantalla HMI KTP 700 BASIC PN de Siemens. Las herramientas de software son FACTORY I/O y TIA PORTAL.

Para la realización de los objetivos mencionados con anterioridad ha sido necesario:

- Diseñar y estudiar el comportamiento de los diferentes elementos que componen cada una de las estaciones, así como las señales de control de los mismos.

- Programar las instrucciones de control que gobernaran cada una de las estaciones acordes a los requisitos de funcionamiento.

- Realizar el conexionado del ordenador personal con el PLC y el HMI para crear una red local a través de la cual puedan comunicarse.

- Estudiar y analizar la documentación de FACTORY I/O para conocer cómo se realiza la comunicación con diferentes elementos tanto de tipo software como hardware.

- Investigar sobre la correcta configuración del hardware de la CPU del autómata para que pueda comunicarse con FACTORY I/O.

- Implementar la comunicación entre FACTORY I/O y el software de simulación de PLCSIM para poder visualizar el comportamiento de las estaciones sin necesidad de disponer de un PLC.

- Programar el algoritmo de control de lazo cerrado PID para controlar el proceso continuo.

- Configurar la pantalla HMI para que se comunique con el PLC y que responda a las órdenes dadas por el operario de control de planta
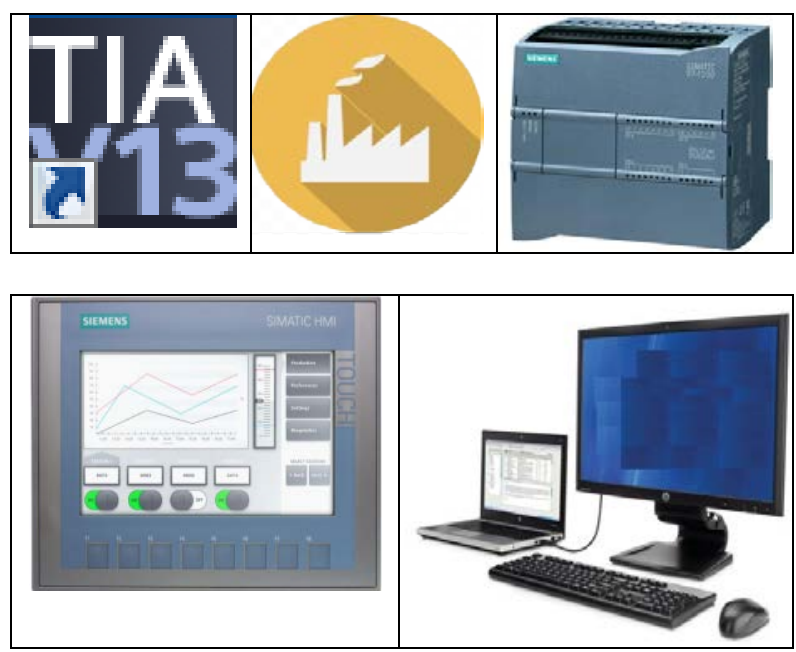

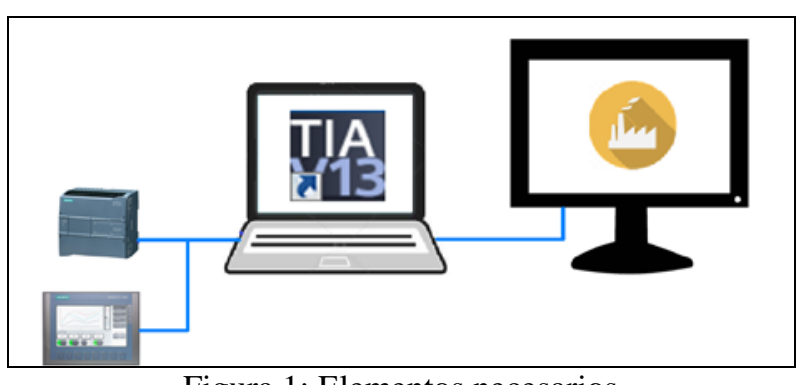

Figura 1: Elementos necesarios

\section{ELEMETOS NECESARIOS}

Los elementos necesarios para realizar este trabajo son, Figura 1:

- Software de programación de PLC y HMI

- Software de simulación Factory I/O

- PLC 1200 de SIEMENS

- Cámara de órbita

- HMI

- Ordenador con pantalla externa

- Cableado para establecer todas las comunicaciones.

\section{METODOLOGÍA}

A continuación, presentamos los pasos que se han seguido en todos los casos realizados

\subsection{INTRODUCCIÓN}

El uso de FACTORY I/O nos permite realizar de forma sencilla simulaciones de fábricas en 3D para el aprendizaje de tecnologías de automatización. Permite construir rápidamente una fábrica virtual utilizando una selección de piezas industriales comunes.

Por otro lado, incluye muchas escenas inspiradas en aplicaciones industriales típicas de diferente nivel de dificultad.

El escenario más común es usar el software como una plataforma de entrenamiento de PLC ya que son los controladores más comunes que se encuentran en aplicaciones industriales. Sin embargo, este programa también ofrece la posibilidad de utilizar microcontroladores, SoftPLC, Modbus, entre otras muchas tecnologías.

\subsection{NAVEGACIÓN}

Se dispone de varias cámaras virtuales que nos permiten navegar en el espacio 3D y son clave para interactuar con las diferentes partes. Las cámaras son de tres tipos diferentes y cada una fue diseñada para un propósito funcional específico. Estos tres tipos de cámaras son: 
- Cámara de órbita

- Cámara Fly

- Cámara de primera persona

\subsection{CONTROL CON PLC}

El control de la escena mediante el uso de un PLC se realiza a través de los controladores de Entrada/Salida que incorpora FACTORY I/O, el cual proporciona una lista de escenas para usar inspiradas en aplicaciones industriales de diferente nivel de dificultad

FACTORY I/O incluye muchos controladores de E/S, cada uno para una tecnología específica. Para el caso de este trabajo se han adquirido la licencia de controladores de la marca SIEMENS.

Configurando adecuadamente dicho controlador, este sabrá leer y escribir Entradas/Salidas desde el mismo.

En la Figura 2, se muestra un esquema de la configuración general de comunicación entre FACTORY I/O y TIA

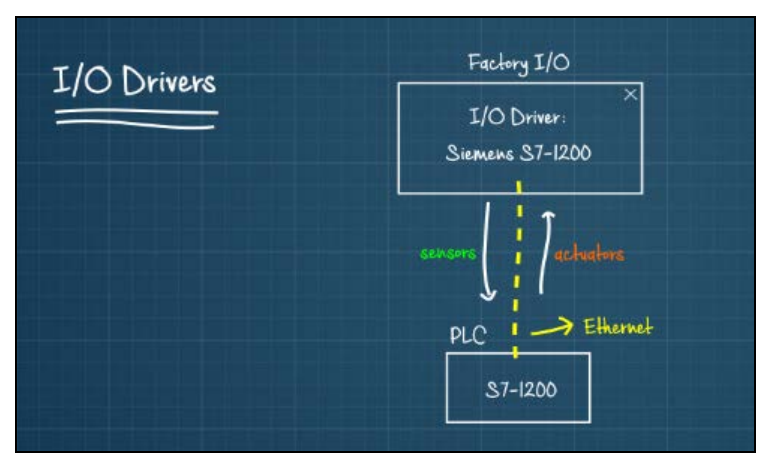

Figura 2: Configuración

\subsection{Componentes}

Se incluye una colección de piezas basadas en el equipo industrial más común. Hay más de 80 partes, organizadas en ocho categorías: artículos, piezas de carga pesada, partes de carga ligera, sensores, operadores, estaciones, dispositivos de aviso y pasarelas, como se ve en la Figura 4.

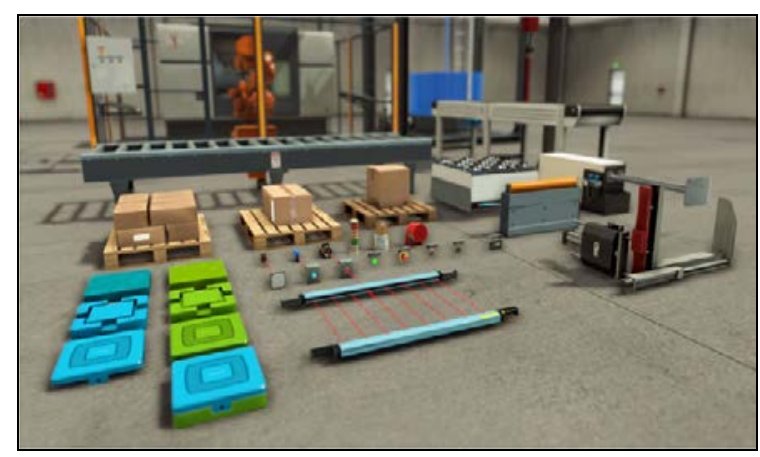

Figura 3: Componentes

\section{ESTACIONES DE TRABAJO}

En este apartado se pasa a presentar cada una de las estaciones de trabajo diseñadas y programadas para cumplir ciertos requisitos de funcionamiento.

Para ello se van a presentar las siguientes estaciones:

- $\quad$ Estación paletizadora, Figura 4.

- $\quad$ Estación distribuidora, Figura 7.

- $\quad$ Estación depósito-líquido, Figura 11.

- Estación gestión almacén stock, Figura 16

Y para cada estación vamos a seguir el mismo esquema general de presentación, el cual consta de:

- Descripción del funcionamiento

- Conexionado de E/S

- Programación del autómata

\subsection{Estación paletizadora}

\subsubsection{Descripción funcionamiento}

En esta estación se realizó la automatización de un proceso de paletizado en el que una serie de cajas son distribuidas de una determinada forma sobre un palé.

La estación consta de una cinta transportadora por donde llegan las cajas que deben ser posicionadas sobre el palé. Al final de la misma, se encuentra un pistón cuya función es posicionar de forma adecuada la caja para que pueda ser accesible por el robot cartesiano.

También hay una cinta de rodillos a través de la cual se van desplazando los palés, en mitad de la cinta se encuentra un rodillo de parada que sirve para impedir el avance del palé cuando ya se encuentra en la posición adecuada.

El elemento que es encargado de llevar a cabo todo el proceso de paletizado de cajas es un robot cartesiano. El robot distribuirá 6 cajas sobre cada palé, de tal forma que, dependiendo del número de caja, el robot se situará sobre unas posiciones u otras.

Cuando un palé está completo se vuelve a poner en marcha la cinta de rodillos, desplazando el palé que ya se encuentra ocupado por cajas hacia el final de la cinta, al mismo tiempo que otro nuevo palé se coloca en la posición correspondiente para ser cargado por cajas.

Para gobernar el comportamiento de la estación por parte del operario se dispone de un cuadro de mando con los siguientes elementos:

- Botón de marcha

- Botón de paro

- Botón de parada de emergencia 


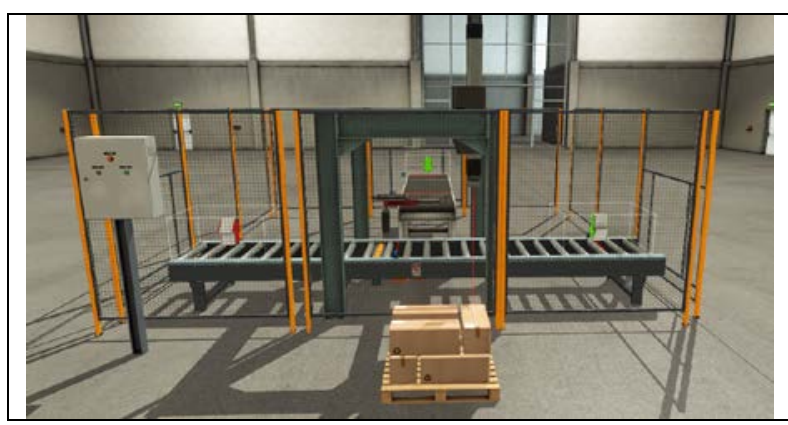

Figura 4: Estación Paletizadora

\subsubsection{Conexionado de entradas/salidas}

Las entradas y salidas utilizadas para realizar el programa en TIA PORTAL son las mismas que las utilizadas en la configuración de la estación en FACTORY I/O ya que, si no se realiza de esta forma, no existe una correcta comunicación entre el autómata y el modelo de simulación.

A continuación, se muestra en una tabla las entradas y salidas utilizadas en la elaboración del programa en TIA PORTAL y como se muestran esas conexiones en el PLC virtual de FACTORY I/O, Figura 5.

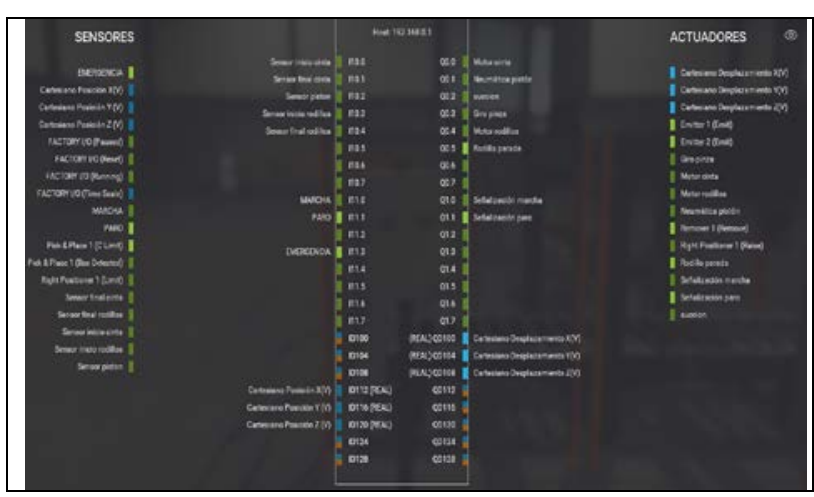

Figura 5: Distribución E/S estación paletizadora

\subsubsection{Programación del autómata}

La programación del autómata se realizar mediante la utilización del lenguaje de contactos o KOP, adquiriendo la siguiente estructura general del programa, Figura 6.

\subsection{Estación distribuidora}

\subsubsection{Descripción funcionamiento}

En esta estación se automatiza de un proceso en el cual dos tipos diferentes de piezas son distribuidas y almacenadas en cajas de cuatro unidades cada una para su posterior transporte.

A ambos lados del clasificador se encuentran dos líneas distribuidoras de producto independientes una de la otra.
Al inicio del proceso se encuentra una cinta transportadora sobre la cual llegan diferentes tipos de piezas. Al final de la misma hay un clasificador de ruedas, al que se le ha incorporado un sensor de visión artificial, de tal forma que si el sensor detecta que la pieza es de un tipo determinado, activa el clasificador desplazando la pieza situada sobre él hacia un lado u otro del mismo.

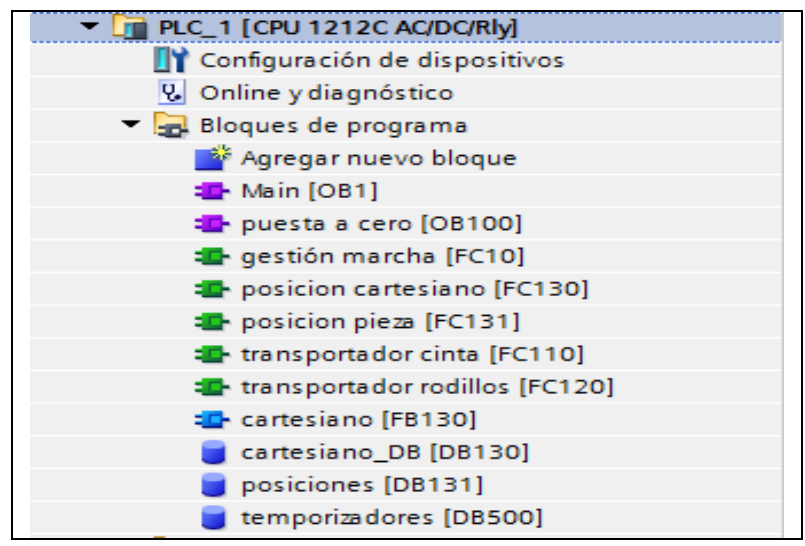

Figura 6: Bloques estación paletizadora

Al final de cada línea se encuentra una barrera de parada que detiene el desplazamiento de la pieza, colocándola en la posición adecuada para que posteriormente pueda ser alcanzada por el robot.

Cada robot tiene asociado otra cinta transportadora sobre la cual aparecerán las cajas en las que se van a almacenar hasta cuatro unidades de piezas de cada tipo. Cuando una caja se ha completado con cuatro piezas del mismo tipo, se desplaza hasta el final de la línea, volviendo otra caja vacía a ocupar la posición abandonada por la caja anterior para ser nuevamente ocupada por piezas.

Para gobernar el comportamiento de la estación se dispone de un cuadro de mando con los siguientes elementos:

- Botón de marcha

- Botón de paro

- Botón de parada de emergencia

- Botón de reset de contadores de piezas

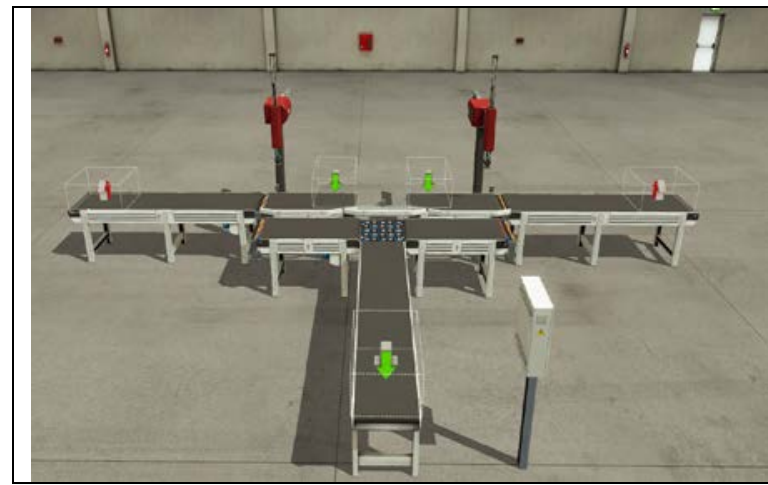

Figura 7: Estación distribuidora 


\subsubsection{Conexionado de entradas/salidas}

A continuación, se muestra en una tabla las entradas y salidas utilizadas en la elaboración del programa en TIA PORTAL y como se muestran esas conexiones en el PLC virtual de FACTORY I/O.

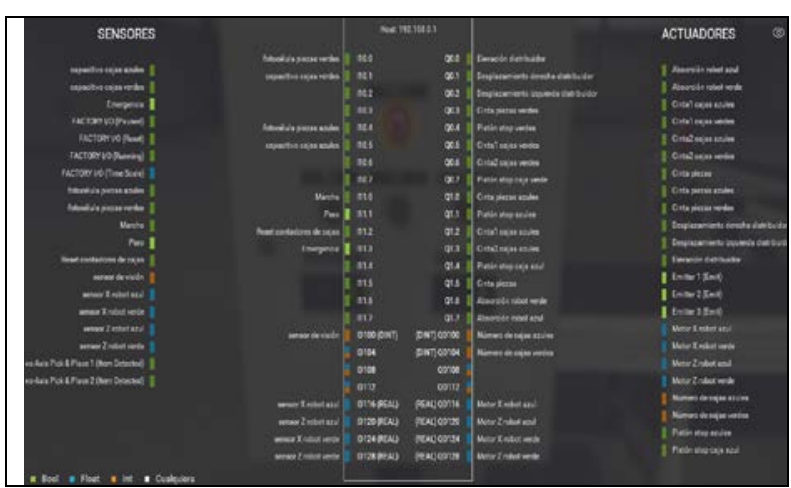

Figura 8: Distribución E/S estación distribuidora

\subsubsection{Programación del autómata}

La programación del autómata se realiza mediante la utilización del lenguaje de contactos o KOP, adquiriendo la siguiente estructura general del programa.

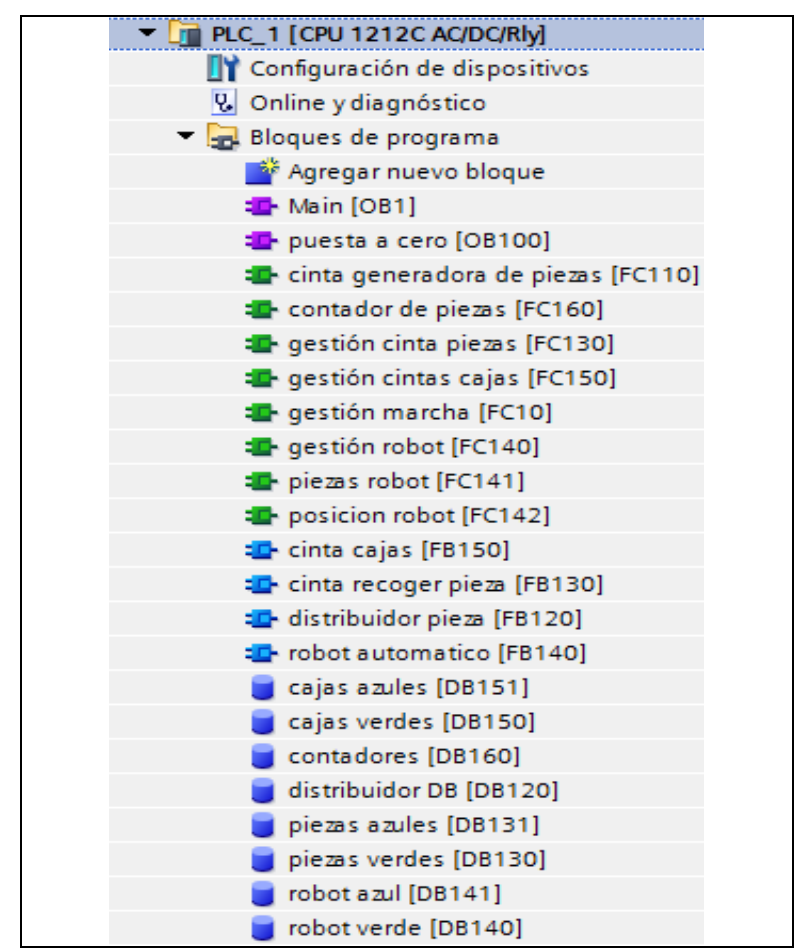

Figura 9: Bloques estación distribuidora

\subsection{Estación depósito líquido}

\subsubsection{Descripción funcionamiento}

En este caso se trata de controlar un proceso continuo que consiste en el llenado y vaciado de un depósito mediante una serie de válvulas.

La estación consta de un depósito de líquido que posee dos válvulas diferentes, una para realizar el llenado del mismo, y otra para vaciarlo. Junto a este depósito se encuentra un cuadro de mando desde el cual el operario puede seleccionar una cantidad determinada de líquido mediante el uso de un potenciómetro y añadirla al depósito o extraerla.

A su vez se han incorporado dos display encargados de informar al operario de la cantidad de litros seleccionados y la cantidad de litros que contiene el depósito en su interior.

Cada válvula del depósito posee su propio controlador PID ya que son elementos independientes uno del otro, Figura 10

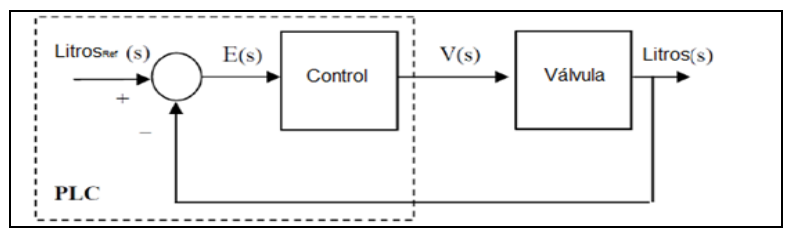

Figura 10: Esquema control PID válvula depósito

En el esquema, E(s) representa la señal de error y es la diferencia entre los litros deseados en el depósito (referencia) y los litros realmente contenidos en su interior. La zona marcada con línea discontinua representa el autómata programable (S7-1200), en el que se encuentran los sistemas de control (PID) y desde el que se generan las señales de referencia.

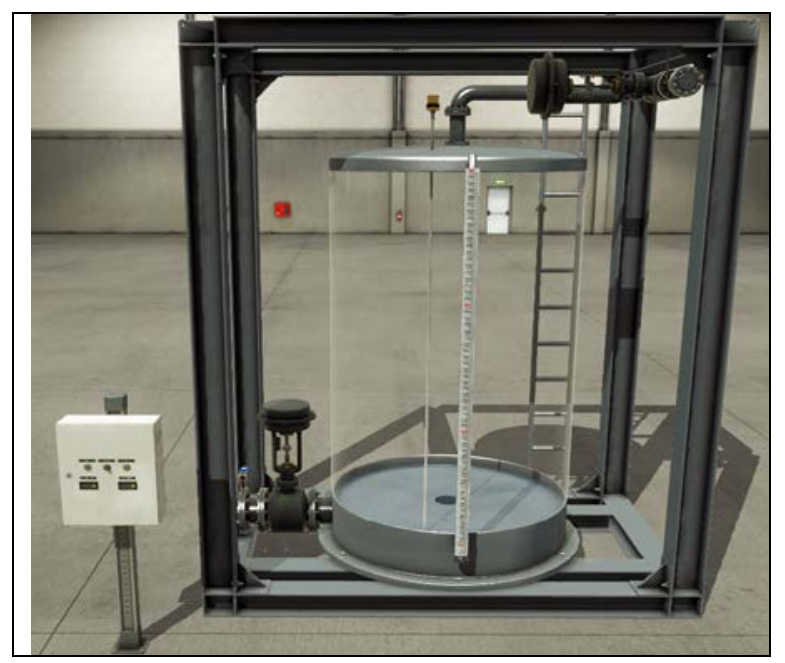

Figura 11. Estación depósito líquido

\subsubsection{Conexionado de entradas/salidas}

A continuación, se muestra en una tabla las entradas y salidas utilizadas en la elaboración del programa en TIA PORTAL y como se muestran esas conexiones en el PLC virtual de FACTORY I/O. 


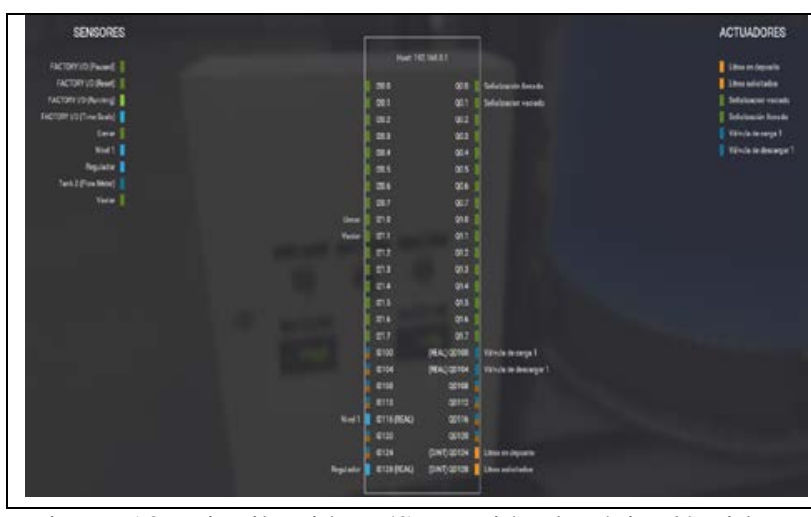

Figura 12: Distribución E/S estación depósito líquido

\subsubsection{Programación del autómata}

La programación del autómata se realiza mediante la utilización del lenguaje de contactos o KOP, adquiriendo la siguiente estructura general del programa.

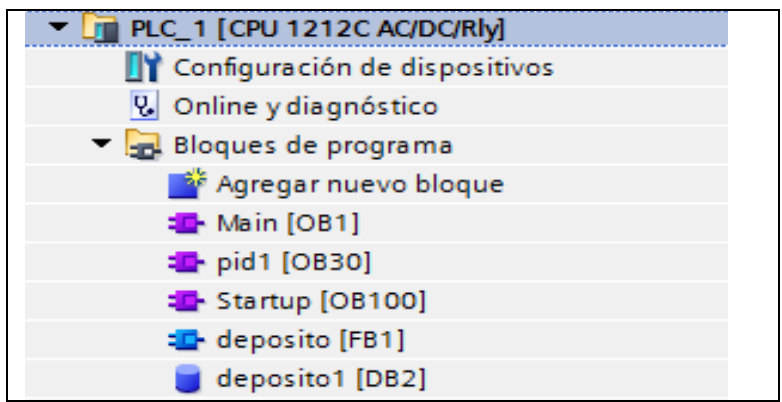

Figura 13: Bloques estación depósito líquido

Para la programación del control PID se utiliza el bloque PID_COMPACT proporcionado por TIA PORTAL configurándolo adecuadamente para que las características de respuesta se adecuen a las exigencias de nuestro sistema.

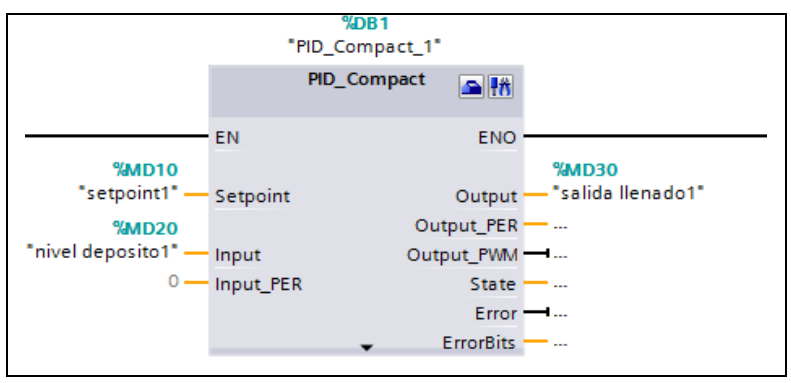

Figura 14: Bloque PID_COMPACT

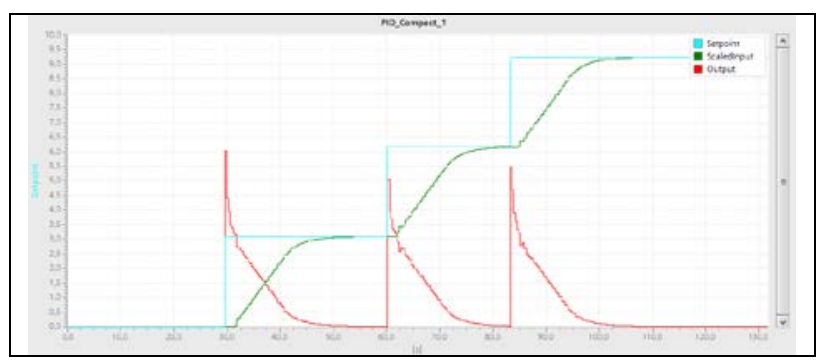

Figura 15: Visualización comportamiento PID

\subsection{Estación gestión de stock almacén}

\subsubsection{Descripción funcionamiento}

Con esta estación se pretende automatizar el stock de un almacén y controlar su gestión mediante una pantalla HMI manipulada por parte del operario.

El funcionamiento básico de la estación consiste en que por una cinta transportadora de rodillos llegan diferentes tipos de piezas en palé. Al final de esta cinta transportadora se encuentra una cámara de visión artificial que nos permite diferenciar un tipo de piezas u otras en función de su color y forma.

Una vez que la pieza ha sido reconocida y el palé se encuentra en la posición adecuada, el carro repartidor de la estación desplazará el palé con la pieza al lugar asociado del almacén, en función del tipo de pieza.

Cuando el palé con la pieza es retirado de la cinta transportadora de entrada, otro nuevo palé con otra pieza se desplazará a dicha posición para ser también almacenado.

Cuando el carro repartidor ha depositado el palé con la pieza en la posición correspondiente del almacén, vuelve a la posición de inicio para volver a repetir el proceso con otro nuevo palé.

Por otro lado, el operario va a disponer de una pantalla HMI para controlar la gestión del almacén. En dicha pantalla, en la imagen principal, se disponen de dos botones uno de MARCHA y otro de PARO para controlar el funcionamiento de la estación. Además, el operario dispone de la opción de seleccionar el tipo de gestión que quiere realizar con los productos que se van almacenando en el almacén. Si prefiere adoptar una gestión de stock tipo FIFO (First Input First Output) o a su vez prefiere una gestión tipo LIFO (Last Input First Output).

En esa misma pantalla el operario dispone a su disposición de un botón que le permite acceder a otra nueva pantalla donde puede seleccionar el tipo de pieza deseada y ver toda su información asociada. Entre esa información se encuentra el número de piezas de ese tipo que hay en stock y el tiempo que llevan en el almacén. Es aquí donde el operario decide si desea extraer algunas de las piezas de ese tipo que se encuentran es stock.

Cuando el operario decide extraer alguna pieza del stock, el carro repartidor de la estación se dirige a la fila del tipo de pieza seleccionada y en función del tipo de gestión (FIFO/LIFO), empezará a extraer piezas en una posición un otra de la fila.

Una vez la pieza ha sido extraída y depositada en el 
carro repartidor, este se dirige a la cinta transportadora de salida donde será depositado el palé y finalmente abandonará la estación.

Cuando el carro repartidor haya terminado de extraer todas las piezas solicitadas por el operario, volverá a reanudar su trabajo colocando los nuevos palés que le van llegando por la cinta transportadora de entrada.

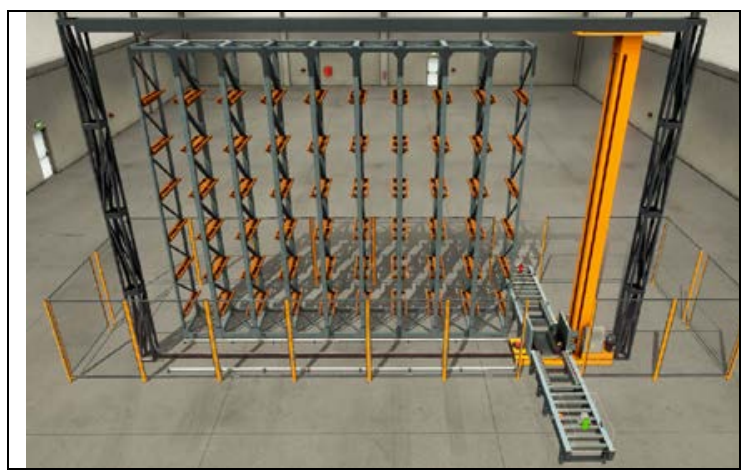

Figura 16: Estación gestión de stock almacén

\subsubsection{Conexionado de entradas/salidas}

A continuación, se muestra en una tabla las entradas y salidas utilizadas en la elaboración del programa en TIA PORTAL y como se muestran esas conexiones en el PLC virtual de FACTORY I/O.

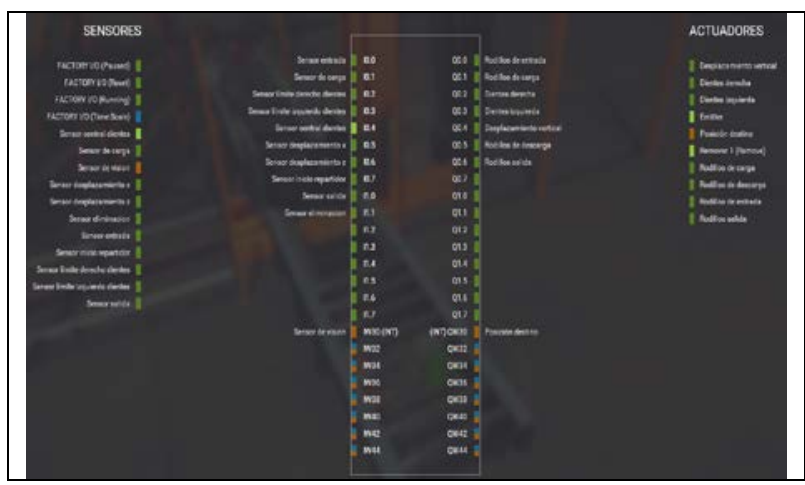

Figura 17 Distribución E/S estación gestión stock

\subsubsection{Programación del autómata}

Como siempre la programación del autómata se realiza mediante la utilización del lenguaje de contactos o KOP, Figura 18. Lo nuevo que aporta esta estación es una pantalla HMI, Figura 20.

\subsubsection{Programación del HMI}

La programación del HMI se realiza mediante la utilización de imágenes a las cuales se las van agregando diferentes tipos de objetos con unas propiedades específicas, estos objetos pueden realizar ciertas acciones sobre las variables del PLC o sobre las propias variables del HMI. La estructura general de las imágenes del HMI es la que se muestra a continuación:

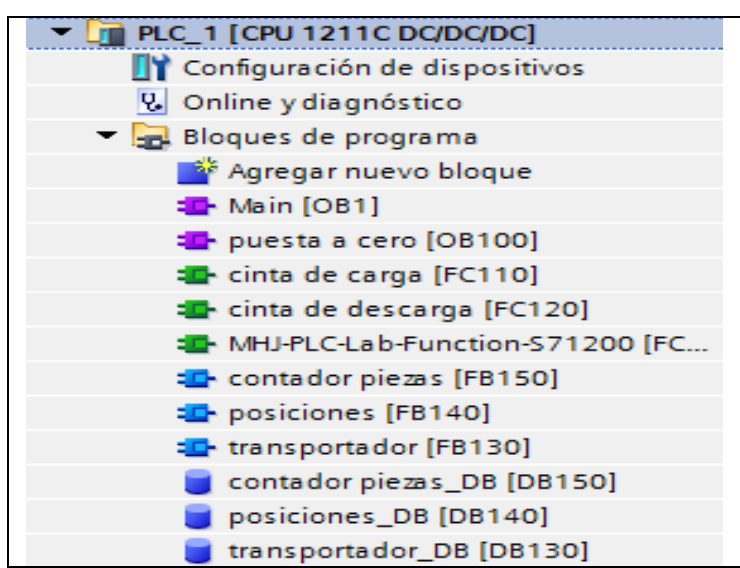

Figura 18: Bloques gestión stock almacén

\begin{tabular}{|l|}
\hline HMI_[KTPZoo Basic PN] \\
$\square$ Configuración de dispositivos \\
Q. Online y diagnóstico \\
Configuración de runtime \\
$\square$ Imágenes \\
$\square$ Agregar imagen \\
$\square$ Base azul \\
$\square$ Imagen raíz \\
$\square$ Prima azul \\
$\square$ Prima verde \\
$\square$ Productos \\
$\square$ Tapa azul \\
$\square$ Tapa verde
\end{tabular}

Figura 19: Bloques HMI

Siendo las siguientes algunas de las imágenes con las que trabaja el operario:

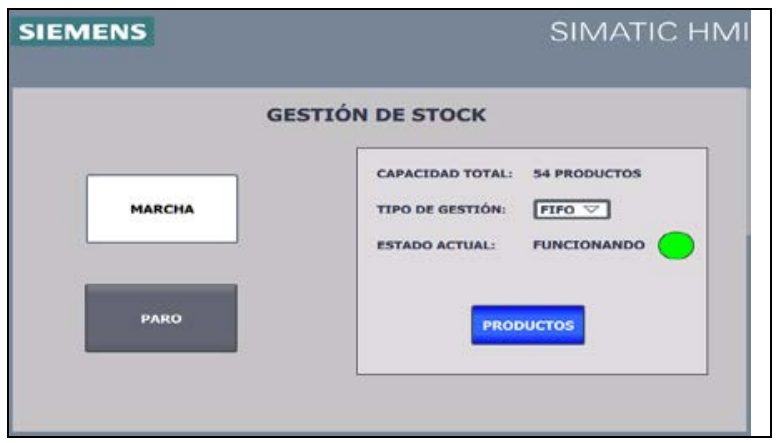

Figura 20: Gestión stock almacén

\section{CONCLUSIONES}

Tras la finalización de la programación y la simulación de las diferentes estaciones presentadas anteriormente y observando los resultados obtenidos podemos establecer que el uso del software FACTORY I/O facilita claramente la adquisición de nuevos conceptos en cuanto a materia de programación de PLC se refiere.

En las estaciones anteriores, a la hora de realizar su programación no se abordaba directamente la programación completa de la misma, sino que se iban dividiendo en secciones y programando poco a poco. 
Cada vez que se tenía una sección programada, se simulaba para ver su comportamiento, lo que en muchos casos permite darse cuenta de la existencia de errores de programación que de otra forma hubieran pasados desapercibidos y sería a la hora de poner en marcha la estación completa cuando hubieran aparecido y siendo mucho más complicado dar con ellos y tratar de corregirlos.

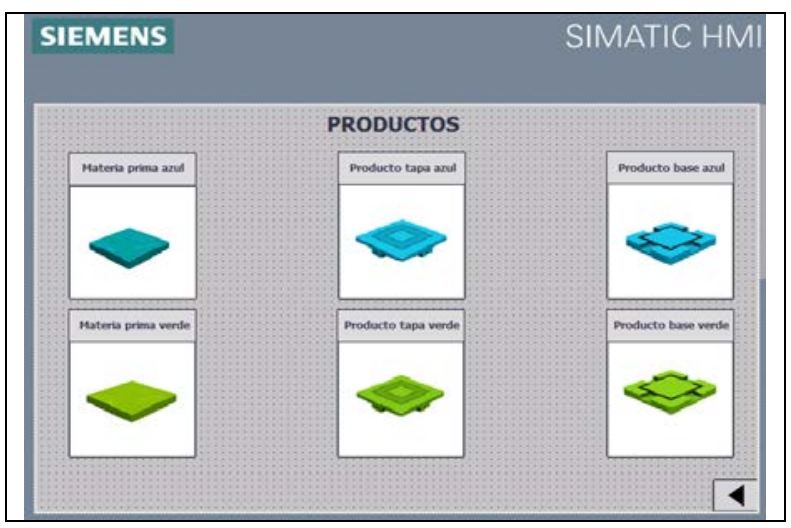

Figura 21: Imagen productos HMI

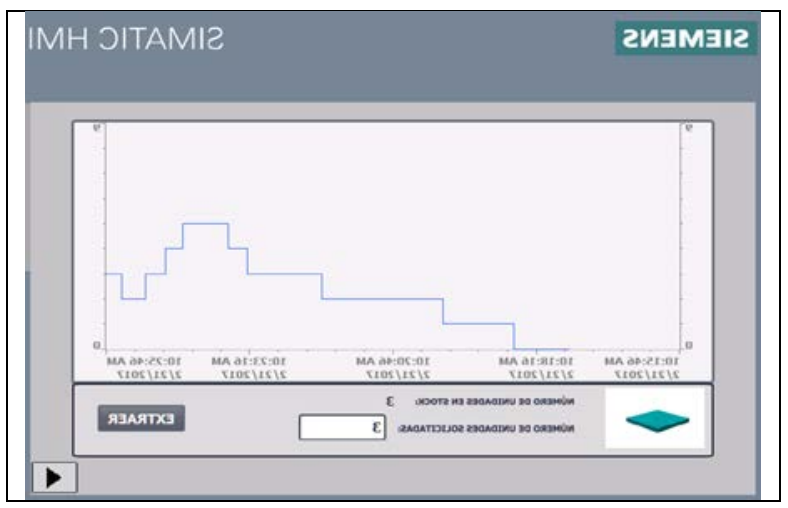

Figura 22: Registro Histórico

Por otro lado, el uso del PLC físico en todas aquellas estaciones cuya simulación se ha realizado mediante el uso del mismo, permite reforzar los conocimientos en el campo de redes de comunicación ya que es necesaria la creación de una red local para la correcta simulación de la estación.

A sí mismo, en el caso concreto del control del depósito del tanque, el uso del elemento de simulación proporcionado por FACTORY I/O permite observar el efecto que tiene sobre dicho elemento físico las diferentes estrategias de control, de otro modo hubiera sido necesario la existencia de un sistema físico con su respectivo coste y mantenimiento para poder observar los resultados.

También se ha observado que se obtienen ventajas en cuanto al uso y la programación de pantallas HMI ya que por un lado nos permite apreciar el efecto inmediato que tiene sobre la estación la activación de algún botón o elemento de la pantalla y por otro lado nos permite hacer uso de elementos proporcionados para la programación de dichas pantallas como el uso de gráficas para monitorizar el comportamiento de ciertos procesos, el uso de avisos de sistema en caso de que se produzca algún error en la estación o el uso de recetas para cambiar ciertas variables del proceso, que de otro modo hubieran sido muy complicados de simular.

Por lo tanto, el software FACTORY I/O es un elemento más que recomendable tanto para todos aquellos que se estén iniciando en el mundo de la automatización industrial como para todas aquellas instituciones que se dediquen a la enseñanza de dicha materia.

Se han diseñado una serie de prácticas adecuadas para la docencia de la asignatura de regulación automática en la Escuela de Ingenieros Industriales de la Universidad de Valladolid.

\section{Referencias}

[1] Controlador S7-1200 manual de sistema.:https://w5.siemens.com/spain/web/es/i ndustry/automatizacion/simatic/Documents/S71 200-MANUAL\%20DEL\%20SISTEMA.PDF (último acceso 17/05/2017)

[2] Programar en STEP 7: https://cache.industry.siemens.com/dl/files/056/ 18652056/att_70833/v1/S7prv54_s.pdf. (último acceso 17/05/2017)

[3] Simatic Step 7 en TIA PORTAL:

https://w5.siemens.com/spain/web/es/industry/a utomatizacion/simatic/Tia-

Portal/tia_portal/Documents/Folleto\%20STEP7 \%20en\%20TIAP\%20Nov11.pdf (último acceso 17/05/2017)

[4] Manual de sistema WINCC BASIC v13.0: https://cache.industry.siemens.com/dl/files/840/ 91379840/att_110005/v1/WinCC_Basic_V13_e sES_es-ES.pdf (último acceso 17/05/2017)

[5] Control PDI con PID_COMPACT: https://cache.industry.siemens.com/dl/files/401/ 100746401/att_15889/v1/100746401_s71200_pid_compact_doku_v1d0_en.pdf (último acceso 17/05/2017)

[6] Manual de instalación de software FACTORY I/O: https://factoryio.com/docs/installing/ (último acceso 17/05/2017)

[7] Configuración de drivers de FACTORY I/O para la gama S7-1200/1500

https://factoryio.com/docs/manual/drivers/s71200-1500/ (último acceso 17/05/2017)

[8] Diseño de elementos de simulación de FACTORY I/O

https:/factoryio.com/docs/manual/parts/ (último acceso 17/05/2017) 